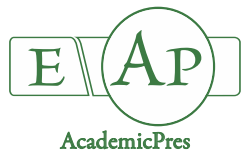

\title{
Identification and Characterization of a SEPALLATA-like MADS-box Gene from Cucumber (Cucumis sativus L.)
}

\author{
Yanwei CHENG ${ }^{1}$, Peng $\mathrm{HE}^{2}$, Lunwei JIANG ${ }^{3}$, Shiqiang LIU ${ }^{3}$, \\ Yong $\mathrm{ZHOU}^{3,4, *}$ \\ ${ }^{1}$ Luoyang Normal University, College of Life Science, Luoyang 471934, China; chengyanwei@lynu.edu.cn \\ 2Jiangxi Agricultural University, College of Agronomy, Nanchang 330045, China; hepeng_17@163.com \\ 3 Jiangxi Agricultural University, College of Bioscience and Bioengineering, Nanchang 330045, \\ China;jlp_1999@163.com;lsq_hn306@163.com;yongzhou@jxau.edu.cn (*corresponding author) \\ ${ }^{4} J$ iangxi Agricultural University, Key Laboratory of Crop Physiology, Ecology and Genetic Breeding, Ministry of Education, Nanchang 330045, \\ China; yongzhou@jxau.edu.cn (*correspondingauthor)
}

\begin{abstract}
SEPALLATA $(S E P)$ genes encode the E class MADS-box transcription factors that play vital roles in various aspects of plant growth and development. In this study, we isolated a $S E P$-like gene from cucumber (Cucumis sativus L.), which was previously named as CsMADSO1. CsMADSO1 had a coding sequence (CDS) of $741 \mathrm{bp}$, and coded a deduced protein of 246 amino acid residues that was predicted to be located in the nucleus. The putative CsMADS01 protein was typically characterized by the MIKC-type MADS (including MADS, I, K, and C domains) and shared high homology with other SEPlike proteins. Phylogenetic analysis of CsMADS01 and SEP proteins from other plants revealed that CsMADS01 was a member of the SEP1/2 clade of SEP proteins and was the most closely related to ZjMADS48 from Chinese jujube. Promoter analysis showed that several cis-elements related to stress response and hormones were present in the promoter region of CsMADS01. Expression analysis indicated that CsMADSO1 was highly expressed during the development of male and female flowers, and the expression increased gradually along with fruit development. Ectopic expression of CsMADSO1 in Arabidopsis resulted in a phyllody-like phenotype and the transgenic plants never flowered. These results suggest that CsMADSO1 plays an important role in the growth and development of cucumber.
\end{abstract}

Keywords: cucumber; gene expression; MADS-box; SEPALLATA (SEP); transgenic Arabidopsis

\section{Introduction}

MADS-box family genes encode transcription factors characterized by the presence of 58-60 highly conserved Nterminal DNA binding domain (termed as the MADS domain), which is conserved across a wide range of organisms including plants, fungi, and mammals (Riechmann and Meyerowitz, 1997; Xu et al., 2014; Nardeli et al., 2018). According to the phylogenetic analysis, the MADS-box genes can be divided into two functional types named as type I and type II (Alvarez-Buylla et al., 2000; Masiero et al., 2011), and the type II members are also termed as MIKC-type MADS for the presence of three additional domains from $\mathrm{N}$-terminal to C-terminal: intervening (I) domain, keratin $(\mathrm{K})$ domain, and $\mathrm{C}$ terminus (C) domain, when compared with the type I members (Kaufmann et al., 2005; Ren et al., 2017; Zhou et al., 2019b). The MIKC-type MADS genes are well-known for their roles in floral organ development. Except for APETALA2 (AP2), all A, B, C, D, and E class genes belong to the MIKC-type MADS-box genes according to the hypothesis of floral organ ABCDE model, and these genes specify the formation of floral organs in a combinatorial way (Weigel and Meyerowitz, 1994; Gutierrez-Cortines and Davies, 2000; Zahn et al., 2006; Theissen et al., 2016).

SEP ALLATA (SEP) genes are the E class genes that are preferentially expressed in flowers and fruits for plant floral organ development (Honma and Goto, 2001; Theissen and Saedler, 2001). The SEP genes were first described in tomato and petunia, and inhibition of their expression would result in highly aberrant flowers (Angenent et al., 1994; Pnueli et al., 1994). Arabidopsis contains four SEP 
genes (SEP1-4), which are expressed in all four whorls of floral organs and play redundant roles in flower meristem identity and organ identity. In sep $1 \operatorname{sep} 2 \operatorname{sep} 3$ triple-mutants, flowers possessed only sepals, while all floral organs were replaced by leaf-like organs in sep 1 sep 2 sep 3 sep 4 quadruple mutants (Pelaz et al., 2000; Ditta et al., 2004; Malcomber and Kellogg, 2005). In addition, the sep1-sep2-sep4 triple mutants displayed a phenotype similar to that of wild-type (WT) plants in floral organ development, revealing that $S E P 3$ is much more critical for flower development than other SEP genes in Arabidopsis (Ditta et al., 2004). Rice contains five floral homeotic genes (OsMADS1, 5, 7, 8 and 34), all of which displayed both functional conservation (E function) and diversification (Cui et al., 2010; Gao et al., 2010; Kobayashi et al., 2012; Khanday et al., 2013; Lin et al., 2014; Meng et al., 2017; Wu et al., 2018). In addition, the SEP proteins can form MADS-box protein complexes with proteins of other classes for floral organ development. For example, OsMADS5 and OsMADS34 can physically interact with candidate class A, B, C, D, E, and AGL6-like floral homeotic proteins to control inflorescence and spikelet morphogenesis (Kobayashi et al., 2012; Hu et al., 2015; Meng et al., 2017; Wu et al., 2018). Besides floral organ development, $S E P$ genes are also involved in many other aspects of growth and development in plants, such as fruit development (Ito et al., 2017; Li et al., 2017), stress response (Chen et al., 2019), bud growth and dormancy (Zhang et al., 2017b). These findings reveal that the E class $S E P$ genes play essential roles in many aspects of plant growth and development with both redundancy and specificity (Soza et al., 2016; Zhang et al., 2017b).

Our previous report has indicated that there are four SEP genes (CsMADSO1-CsMADSO4) in cucumber genome (Hu and Liu, 2012), while only CsMADSO2 was cloned and functionally characterized (Zhou et al., 2019a). In the present study, another SEP gene (CsMADSOI) was isolated from cucumber and its expression profile during flower and fruit development was examined. In addition, a phyllody-like phenotype was observed in the CsMADSO1overexpressing Arabidopsis plants. These findings suggest that CsMADSO1 plays an important role in the growth and development of cucumber.

\section{Materials and Methods}

\section{Plant materials and growth conditions}

Cucumber (Cucumis sativus var. sativus line 9930) and Arabidopsis thaliana ecotypes Columbia-0 (Col-0) were used in this study. Cucumber plants were planted in the field of Jiangxi Agricultural University, Nanchang, China. Different developmental stages of female flowers (MF) and male flowers $(\mathrm{FF})$ were collected for expression profile analysis according to our previous study (Zhou et al., 2019b). The wild-type (WT) and transgenic Arabidopsis seeds were germinated on one-half Murashige and Skoog medium (1/2 MS) and the seedlings were grown on a plant growth chamber in a long-day photoperiod (16-h light/8-h dark cycle) at $22-24^{\circ} \mathrm{C}$.
$R N A$ extraction and cloning of the CsMADSO1 gene

Total RNA was extracted from cucumber female flowers with the TransZol Reagent kit (TransGen, China) following the manufacturer's protocol. After checking the integrity with a Nanodrop 2000 spectrophotometer (Thermo Fisher Scientific, USA), the first-strand cDNA was synthesized with TransScript All-in-One First-Strand cDNA Synthesis SuperMix for qPCR (One-Step gDNA Removal) (TransGen, China). The semi-quantitative reverse transcription PCR (RT-PCR) was carried out to amplify the coding sequence (CDS) of the CsMADSOI gene by using the specific primers CsMADS01-1F (5'aaaCCATGGATGGGAAGAGGAAGAGTAG-3') and CsMADS01-1R (5'aaaAGATCTTCAAAGCATCCAACCAGGGAG-3') based on the sequence of CsMADSOI (Gene ID: Csa004117) $(\mathrm{Hu}$ and Liu, 2012), with the procedure described in our previous study (Zhou et al., 2019b). PCR products were cloned into the $\mathrm{pMD} 18-\mathrm{T}$ vector (TaKaRa, Japan) and sequenced (Shanghai Sangon, China).

\section{Bioinformatics analysis}

The putative CsMADS01 protein sequence was uploaded to ProtParam (http:// web. expasy. org/ protparam/) to calculate the theoretical molecular weight $(\mathrm{MW})$, isoelectric point (pI), and grand average of hydropathy index (GRAVY). The SOPMA tool (https://npsa-prabi.ibcp.fr/cgi-bin/npsa_automat.pl?page = npsa_sopma.html) was used to analyze the secondary structure of CsMADS01. The subcellular localization of CsMADS01 was predicted using the online servers ProtComp 9.0 (http://linux1.softberry.com/berry.phtml), and WoLF PSORT (https:// www.genscript. com/tools/ wolf-psort). The amino acid sequences of CsMADS01 and other selected MADS-box proteins from various plant species were aligned by Clustal Omega (https:// www.ebi.ac.uk/Tools/msa/clustalo/), and the alignments were used to create a neighbor-joining $(\mathrm{NJ})$ phylogenetic tree with the MEGA 7.0 software. The bootstrap was set as 1000 replicates. To predict cis-elements in promoter of CsMADS01, the 1,500 bp regions upstream of the initiation codon (ATG) of CsMADSO1 was examined by the PlantCARE database (http:// bioinformatics. psb. ugent. be/webtools/plantcare/html/).

\section{Gene expression analysis}

RT-PCR was performed to examine the expression of CsMADSO1 in different developmental stages of MF and FF using the reaction procedure previously described (Zhou et al., 2019a). The primer sequences are listed in Table S1. For expression analysis of CsMADSO1 during fruit development, the RPKM values of CsMADSO1 were obtained from the fruitENCODE database (http://www.epigenome.cuhk.edu.hk/ZhongWeb/cucumb er/cucumber_index.jsp) as described previously (Lü et al., 2018).

Vector construction, Arabidopsis transformation and phenotypic analysis

The CDS of CsMADS01 in pMD18-T vector was 
1170

digested with the restriction sites $N c o$ I and $B g l$ II, and ligated into the pCAMBIA1301 vector to generate the overexpression construct pCAMBIA1301-CsMADS01. Then, the resulting plasmid was introduced into Agrobacterium tumefaciens GV3101, and GV3101 containing pCAMBIA1301-CsMADS01 was transformed into Col-0 via the floral dip method (Clough and Bent, 1998). The transgenic Arabidopsis seeds were screened by growing on $1 / 2 \mathrm{MS}$ medium supplemented with $50 \mathrm{mg} / \mathrm{L}$ hygromycin, and transferred to soil. The transcription levels of CsMADSO1 were examined in the transgenic plants by RT-PCR, and the AtTubulin4 gene was used as the internal control, with the procedure described previously (Zhou et al., 2018). The primer sequences are listed in Table $S 1 . \mathrm{T}_{3}$ homozygous offspring were employed for phenotypic analysis.

\section{Results and Discussion}

Cloning and sequence analysis of the CsMADSO1 gene

RT-PCR was used to amplify the ORF of CsMADSO1 gene using specific primers based on the sequence of the CsMADSO1 gene (Gene ID: Csa004117) in our previous study ( $\mathrm{Hu}$ and Liu, 2012). The gene contained the sequence of the complete CDS (741 bp), which encoded a putative protein of 246 amino acids (Fig. 1A). The SMART analysis showed that CsMADS01 possessed the representative MADS and K-box domains, which were located in the positions of 1-60 and 80-172 amino acids, respectively (Fig. 1A). The secondary structure analysis by SOPMA revealed that CsMADS01 possessed 50.41\% alpha helices, $9.35 \%$ extended strands, $5.28 \%$ beta turns, and $34.96 \%$ random coils, respectively (Fig. 1B). In addition, ProtParam analysis showed that CsMADS01 had a theoretical MW of 28.22 $\mathrm{kDa}$, a pI of 9.20, and a GRAVY value of -0.628 , suggesting that it is a hydrophilic and basic protein. Finally, ProtComp and WoLF PSORT results revealed that CsMADS01 was localized to the nucleus. These results indicated that CsMADSO1 encodes a MADS-box transcription factor.

\section{Characterization of CSMADSOI}

BLAST search showed that CsMADS01 had the highest homology to Csa4G126990 in cucumber. It should be noted that the amino acid sequence of Csa4G126990 was identical to the positions of 63-246 amino acids of CsMADS01, and the positions of 1-60 of CsMADS01 was the MADS-box domain (Figs. 1 and 2), which is an indispensable domain for SEP members. Coincidentally, the amino acid sequence of CsMADS01 was identical to the corrected sequence of Csa4G126990, which was formally named as CsSEP2 (Wang et al., 2016). In addition, the CDS and gDNA sequences of CsMADSO1 were also identical to the full sequences of CSSEP2 (Table S2) (Wang

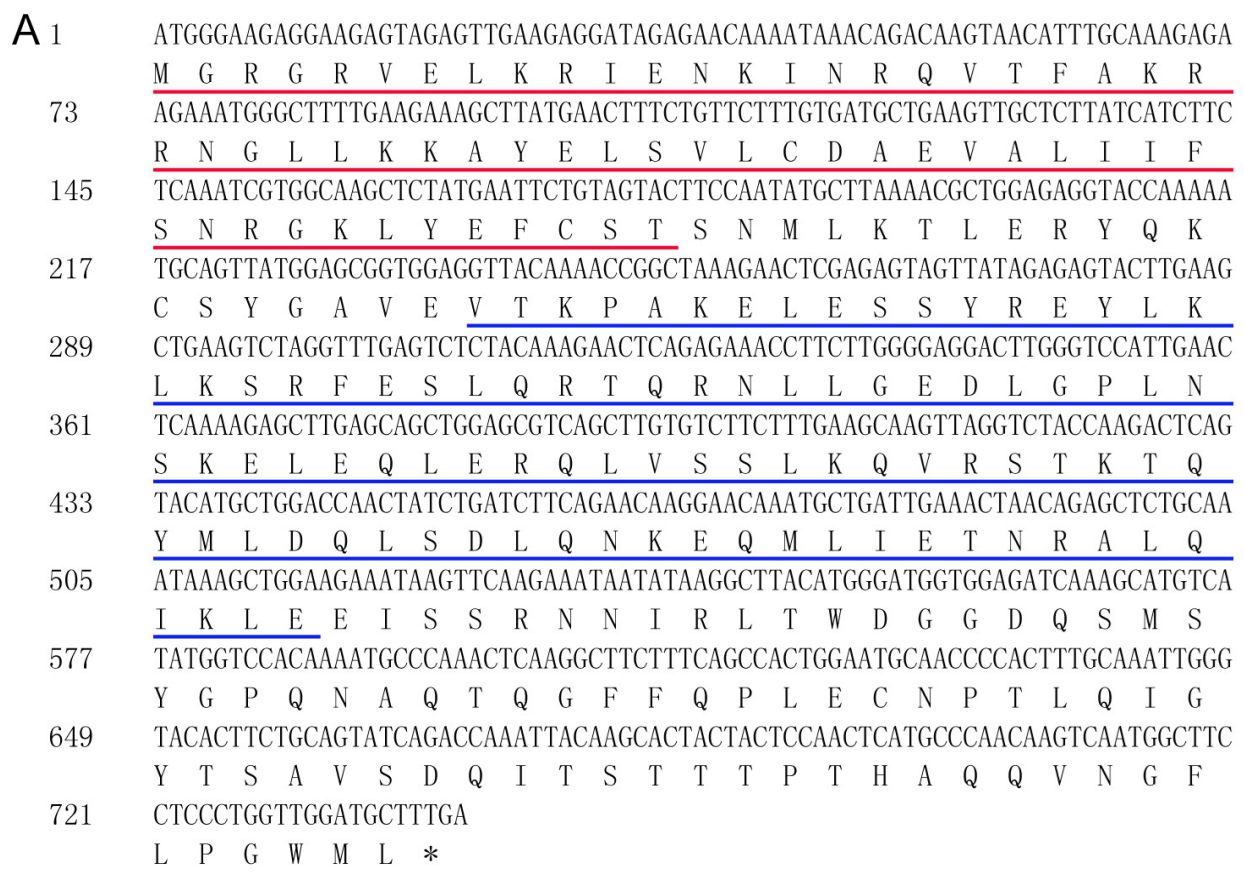

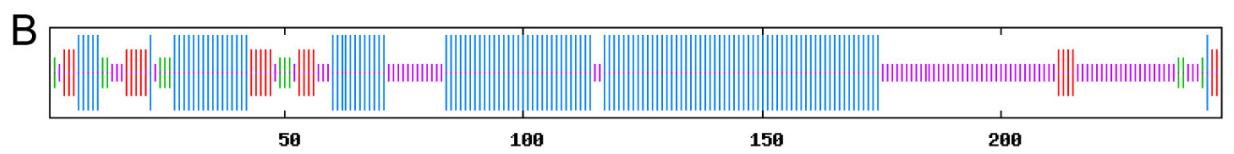

Fig. 1. Sequence analysis of CsMADSO1 and its deduced protein. (A) The CDS and deduced amino acid sequences of CsMADS01. Asterisk represents stop codon. The MADS and K-box domains are underlined with red and blue, respectively. (B) Secondary structure analysis of CsMADS01 by SOPMA. The alpha helix, extended strand, beta turn, and random coil residues are presented by blue, red, green, and purple vertical lines, respectively 
et al., 2016). These results demonstrated that CsMADS01 is identical to CsSEP2, which may be a functional SEP in cucumber.

Our previous study has shown that CsMADS01 is a member of SEP subfamily (Hu and Liu, 2012). We then compared the amino acid sequence of CsMADS01 with that of other SEP proteins in literature references by using Clustal Omega. The amino acid sequence of CsMADS01 showed 53.68-85.19\% identity with these SEP proteins, such as OsMADS34 in rice (53.68\% identity) (Gao et al., 2010; Kobayashi et al., 2010), GbSEP in Ginkgo biloba (55.00\% identity) (Cheng et al., 2016), CaMADS in cucumber $(60.68 \%$ identity) (Chen et al., 2019), CsMADS02 in cucumber $(69.70 \%$ identity) (Zhou et al., 2019a), AtSEP2 in Arabidopsis (74.07\% identity) (Pelaz et al., 2000), BolMADS5 in Brassica oleracea (76.33\% identity) (Sheng et al., 2019), AtSEP1 in Arabidopsis (76.45\% identity) (Pelaz et al., 2001), FaMADS9 in strawberry (80.74\% identity) (Seymour et al., 2011), MdMADS8 in apple (81.48\% identity) (Ireland et al., 2013), ZjMADS48 in Chinese jujube (82.32\% identity) (Zhang et al., 2017a), and PrupeSEP1/PrpMADS7 in peach (85.19\% identity) (Xu et al., 2008; Li et al., 2017). In addition, all the SEP proteins contained a highly conserved MADS domain, followed by less conversed $\mathrm{I}$ and $\mathrm{K}$ domains, and a highly variable $\mathrm{C}$ domain (Fig. 2), which is the characteristic of the MIKC ${ }^{\mathrm{C}}$-group of Type II MADSbox proteins (Ren et al., 2017; Zhou et al., 2017). Moreover, two additional conserved motifs, SEP I and SEP II, were present in the C-terminus of these proteins (Fig. 2), which is typical for SEPs and was also found in other SEP proteins, such as RanSEP3 (Soza et al., 2016), and HrSEP-2 (Mitoma and Kanno, 2018).

\section{Phylogenetic analysis of CSMADSO1 and other SEP subfamily members}

Several gene duplications might have occurred in the most recent common ancestor of angiosperms and gymnosperms, resulting in the division of the SEP-like genes of angiosperms into two major clades, the LOFSEP clade (also called $S E P 1 / 2 / 4$ clade or $A G L 2 / 3 / 4$ clade) and the SEP3 clade, within the SEP phylogeny (Malcomber and Kellogg, 2005; Zahn et al., 2005). To study the phylogenetic relationships of CsMADS01 and other SEP subfamily members, a phylogenetic tree was created based on the amino acid sequences of CsMADS01, CsMADS02 (Zhou et al., 2019a), and SEP proteins from Arabidopsis (Pelaz et al., 2000), rice (Arora et al., 2007), Chinese jujube (Zhang et al., 2017a), and Brassica oleracea (Sheng et al., 2019). These SEP proteins can be clustered into three clades, namely SEP1/2, SEP3, and SEP4 (Fig. 3), which is consistent with the evolution lines reported in previous studies (Xu et al., 2008; Ireland et al., 2013; Zhou et al., 2017; Zhou et al., 2019a). CsMADS01 shared high genetic homology with ZjMADS48, AtSEP1, AtSEP2, and BolMADS5 in the SEP1/2 clade (Fig. 3), suggesting that they may have similar functions.

\section{Cis-element analysis of the CsMADSO1 promoter}

To investigate the possible regulatory functions of the CsMADSO1 gene, the 1,500 bp region upstream of the translational initiation codon of $C S M A D S O 1$ was analyzed. As a result, three kinds of hormone-related cis-elements, such as CGTCA-motif, ABRE, and TCA-element, which are involved in response to methyl jasmonate (MeJA), abscisic acid (ABA), and salicylic acid (SA), respectively, were identified in the CsMADSO1 promoter. In addition, four kinds of stress-related cis-elements were present in the CsMADSO1 promoter, such as $\mathrm{W}$-box response to fungal elicitor, MBS response to drought and salt stress, ARE response to anaerobic induction. These results indicated that CsMADSO1 plays possible roles in hormone and stress responses. Recently, a $S E P$ gene in pepper, CaMADS, was found to act as a positive stress-responsive regulator in the cold, salt, and osmotic stress signaling pathways (Chen et al., 2019).

\section{Expression analysis of CsMADSO1 during flower and fruit} development

Previous results have shown that CSMADSOI is primarily expressed in flowers ( $\mathrm{Hu}$ and Liu, 2012; Wang et al., 2016). To investigate the possible function of CsMADSO1 in flower development, RT-PCR was used to examine the expression of CsMADSO1 in five different developmental stages of MF and FF as described in a previous study (Zhou et al., 2019b). As shown in Fig. 5A and Fig. 5B, CsMADSO1 gene was continuously and highly expressed during the development of male and female

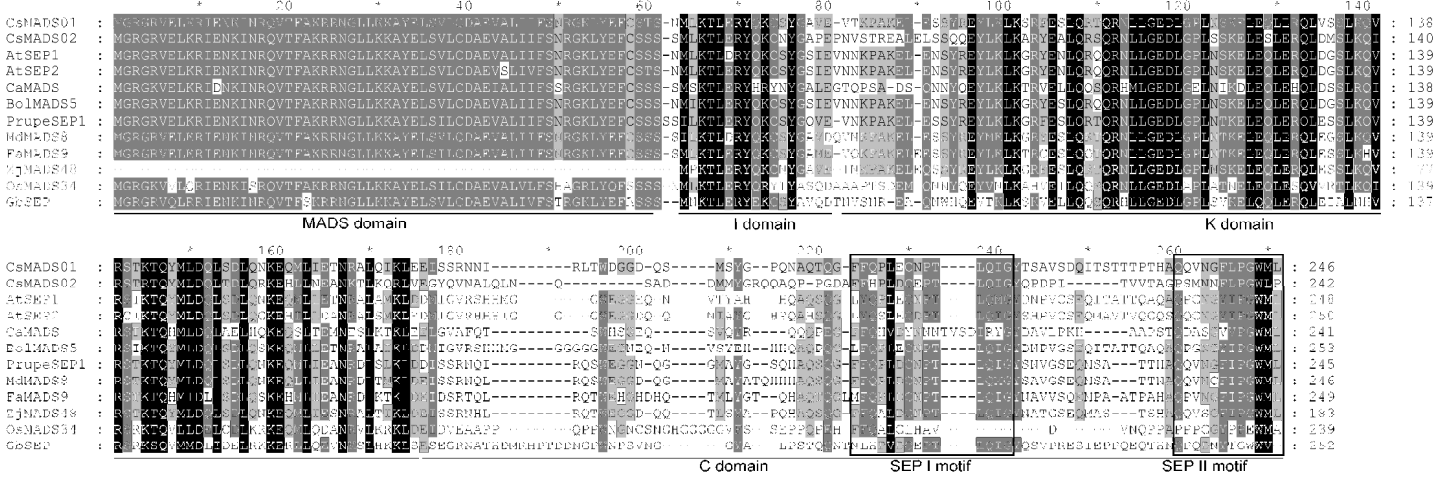

Fig. 2. Multiple sequence alignment of CsMADS01 with SEP proteins from other plant species. The MADS, I, K, and C domains are underlined. Two conserved motifs, SEP I and SEP II, are boxed 
1172

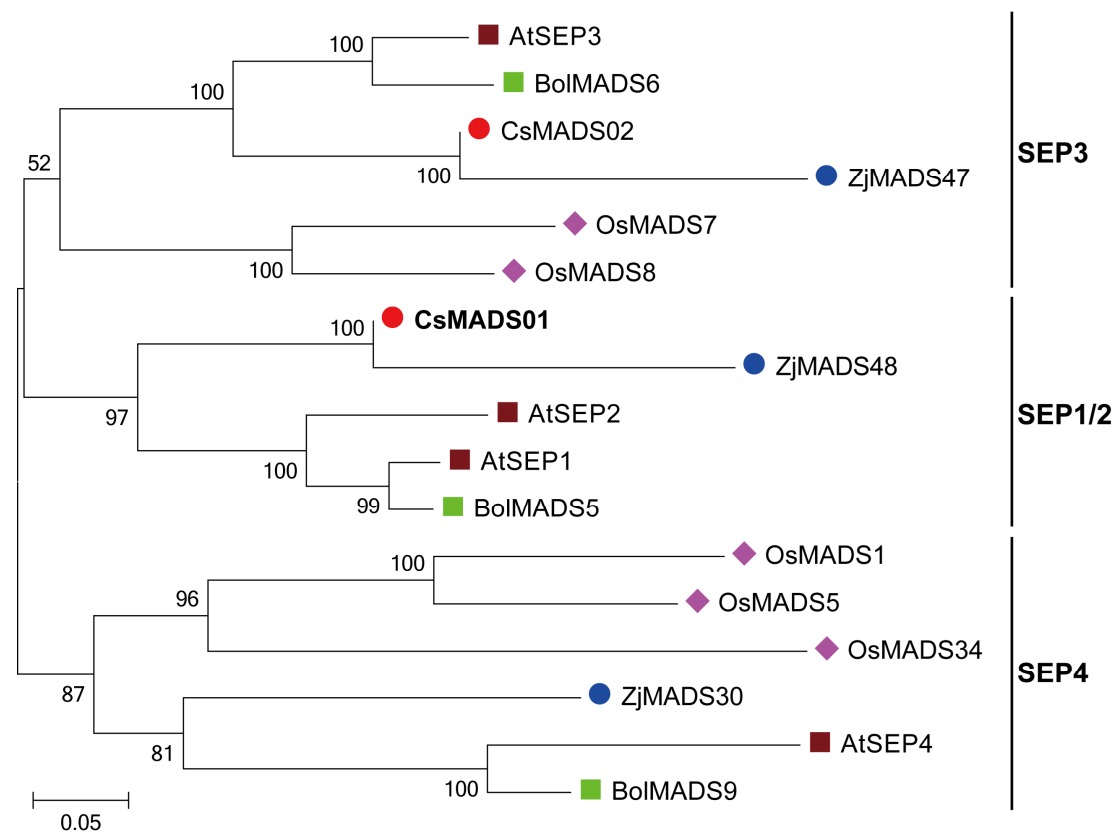

Fig. 3. Phylogenetic analysis of CsMADS01 and other SEP subfamily members from different plant species. Different SEP proteins from cucumber (red round, Cs), Arabidopsis (brown square, At), rice (purple rhombus, Os), and Brassica oleracea (green square, Bol) were aligned with Clustal Omega and the phylogenetic tree was created using the NJ method in MEGA 7.0 by bootstrap analysis with 1000 replicates. CsMADS01 is bolded

\begin{tabular}{|c|c|c|c|c|c|c|}
\hline & AAATAAAAAG & AGGAGAGAAT & TTCAATTTTT & AAAACTATCT & ААСАТТTCTA & ATTAAAATGT \\
\hline 61 & ТТАААСТСТС & AАTGTTAATT & TACAAGTTTA & GTATGAGTTT & ATATTAAACT & TTATTTAGTA \\
\hline 21 & GTTTTCATAG & TTCСAATTTT & ATGTTTGTAC & TCTTTAGGTC & $\begin{array}{l}\text { AAACCTTACA } \\
\text { OX }\end{array}$ & TATTGACTTT \\
\hline 81 & ТСCСАAСТАА & TTTATTGACT & GCTATAGGCT & TTTGAGGGCT & TAATAATTTC & GTTTTTAATG \\
\hline 11 & TCAATAAATA & GGATTTTTCA & ATAAAAGAAA & AAGATAACCT & AAAGATGATT & AAGGAGGCTA \\
\hline 01 & ACCCAGGAAC & ATAGAAACTA & AAAAT & TGAAGTCATA & CTATATAGAT & TAAATAAATA \\
\hline 51 & AATAAAAAGC & AGATCCAGAT & TTAGGAAAAT & CAAAATGTGT & AGGTAACTCT & TTTAAATGAA \\
\hline 21 & AGGAGATGAA & AGTTTGATGA & GAAGTGAATA & TGTTACAAGA & GTTGTTTTAG & AGGAATTAAT \\
\hline 81 & AAGAAGGAAA & GAATGGTATC & AAGTT & TTGGGAATAG & GTATA & TATAAA \\
\hline 11 & AGA & TGGG & AAT & AAAA & GAA & AAA \\
\hline 1 & GTTGGGTA & TAAATAAAAT & TTТTTAAAAA & AAAGGAAGAG & TAGTTAGACG & AATAAGAAAA \\
\hline 61 & GGACAGGTGG & AGATACGTTT & TAAGGTTGOT & GACGACGTTG & GGATGGATCT & GTGATGGGGC \\
\hline 21 & TTTAAGCTGA & TTGATACAAC & TGAGAA & $\begin{array}{l}\text { CA-motif } \\
\text { CCAAATGGGT }\end{array}$ & GTGTGATTTT & AAAAGGTTTG \\
\hline & $\begin{array}{l}\text { AGAAGGCAAT } \\
\text { ABRE }\end{array}$ & CCCACGCGGA & GGGCAGCATA & AGAGAAGGTG & GTGACCAACT & \\
\hline 841 & $\frac{\text { CAdGIGGAAA }}{\text { ABRE }}$ & TGGAGATAAA & AATATCATGT & GGTTATCGAA & TACAATGTGA & $\begin{array}{l}\text { ACGTG TACCA } \\
\text { ABRE }\end{array}$ \\
\hline 01 & CACGCGTGAC & ACAAGGCGTG & AACGAAGCAT & CTTAGGGAAA & AGGAAAAAAG & AAAAACCAGG \\
\hline & & TCAA & & & & \\
\hline 1021 & TTCTG & AGATTGGAAA & ТТTТСААСТT & СТТАAАACCC & АAАCAAACCC & AAACAAACAA \\
\hline 1081 & CAACAGCAAG & AAAGAAAGAA & AGAAAGAAAG & AGAGAAAGAG & AGAAAGAGAG & AAACAAAACC \\
\hline 14 & AACCA & ССССССАAАА & & & & \\
\hline 120 & TTATT & TTTT & TTT & TGAAA & AAC & AGAGTATCAC \\
\hline 1261 & AAAGT & ACACTTGGAG & GGTA & GAGGAAAGAA & AAGAGGGATA & ATCAAGAAGG \\
\hline 1321 & AAGAAAGGAT & TTTTGGGGTA & AGAAAAAAAA & AACAAATAAG & AAAGGGAGTT & TGGAGATTTA \\
\hline 1381 & GGGTAAATAA & $\begin{array}{r}\text { TC-ri } \\
\text { TTGAAAGAGG }\end{array}$ & & GTTGGAGGGG & TGGGGATTTT & TTTATTGACA \\
\hline 1441 & AAAAAGAACA & AAAAGAAGGA & AAGAAAAAAG & GAAAAAGGAA & AGAAAAGAAA & AAAAAAAAGA \\
\hline
\end{tabular}

Fig. 4. Putative cis-element analysis of the CsMADS01 promoter 
flowers, suggesting that it may play an essential role in flower development of cucumber. Similarly, all three SEP subfamily genes in Chinese jujube (ZjMADS30, $Z j M A D S 47$ and $Z j M A D S 48)$ were highly expressed in the sepal, petal and pistil (Zhang et al., 2017a). In mulberry (Morus notabilis), all four SEP subfamily genes also displayed floral organ specific expression (Luo et al., 2018). Two SEP subfamily genes in B. oleracea, BolMADS5 and BolMADS6, also exhibited relatively high expression in four whorls of the flower including the sepal, petal, stamen, and pistil, while another SEP subfamily gene (BolMADS9) was rarely expressed in these tissues (Sheng et al., 2019). Our previous study also revealed that CsMADSO2, another SEP gene in cucumber, also displayed the highest expression in flowers, especially female flowers (Zhou et al., 2019a). However, the expression pattern of CsMADSO2 during female and male flower development was different from that of CSMADSO1 (Figs. $5 \mathrm{~A}$ and $5 \mathrm{~B}$ ). These results indicated that CsMADSO1 and CsMADSO2 may have different functions during flower development in cucumber. This phenomenon may due to the different evolution lines of the two SEP genes: CSMADSO1 and CsMADSO2 are members of the SEP1/2 and SEP3 evolution lines, respectively (Fig. 3). We also examined the expression of CsMADSO1 during fruit development using the transcriptome data from the fruitENCODE database. As shown in Fig. 5C, the expression of CsMADSO1 increased gradually from $10 \mathrm{DPA}$ to $40 \mathrm{DPA}$ during fruit development, while no expression was detected in the leaves. An exon skipping in the CsMADSO1/CsSEP2 gene resulted in defective fruits and enlarged sepals in both female and male flowers (Wang et al., 2016). These results indicated that CsMADSO1 may also function in fruit ripening. Similarly, two apple SEP1/2 evolution line genes $M d M A D S 8$ and $M d M A D S 9$ exhibited higher expression during fruit maturation, and play important roles in both fleshy fruit development and fruit ripening (Ireland et al., 2013). PrupeSEP1 was also found to be involved in the regulation of ripening and softening of melting flesh peach (Li et al., 2017). A tomato SEP gene, MADS-RIN (or RIN), plays vital roles in regulating various aspects of fruit development and ripening through controlling many important ripening genes (Ito et al., 2017; Li et al., 2018; Li et al., 2019).

Overexpression of CsMADSO1 caused a phyllody-like phenotype of transgenic Arabidopsis

To elucidate the potential functions of CsMADSO1, the gene was overexpressed in Arabidopsis under the control of the cauliflower mosaic virus (CaMV) $35 \mathrm{~S}$ promoter. After transformation of Arabidopsis, most transgenic plants displayed significantly altered phenotypes, and two independent transgenic lines with higher expression of CsMADS01, named as OE1 and OE2 (Fig. 6A), were used for phenotypic analysis. The transgenic plants displayed increased numbers of leaves coupled with increased formation of branches, leading to a leafy phenotype (Fig. 6B and 6C). Besides, the phenotype of high-expression transgenic line (OE2) was more severe than that of low- expression transgenic line (OE1), suggesting that the leafy phenotype was due to the ectopic expression of CsMADSO1 in Arabidopsis. Similarly, altered leaf phenotypes were also reported for the ectopic expression of SEP genes in Arabidopsis, such as LMADS3 (Tzeng et al., 2003), TaMADS1 (Zhao et al., 2006), and CsMADSO2 (Zhou et al., 2019a). In addition, the transgenic plants never flowered, while the WT plants could flower normally (Fig. 6D), indicating the flower organs were replaced by leaves in the transgenic plants, resulted in a phyllody-like phenotype. In Arabidopsis, all the four SEP genes play vital roles in specifying the identities of four whorls of the flower and determine floral meristem identity (Pelaz et al., 2000; Ditta et al., 2004; Malcomber and Kellogg, 2005). Single SEP gene mutations resulted in only subtle or no changes in phenotypes when compared with the WT, while floral organs were replaced by leaf-like organs in the sep1 sep 2 sep3 sep4 quadruple mutant (Ditta et al., 2004). A phytoplasmal secreted protein PHYL1 interacts with and degrades the floral homeotic MADS domain proteins APETALA1 (AP1) and SEP1-4 proteins, resulting in the transformation of flower organs into leaf-like structures (Maejima et al., 2014; Maejima et al., 2015). In rice, OsMADS34 is also involved in the transition from vegetative to reproductive growth via the specification of inflorescence meristem identity (Gao et al., 2010), and can act as a transcription

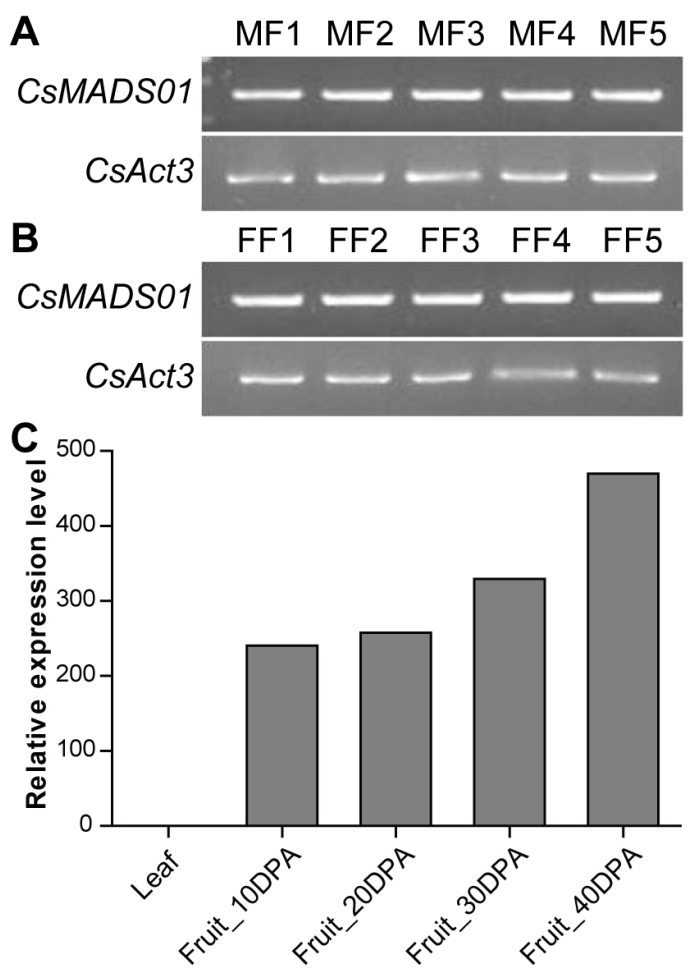

Fig. 5. Expression analysis of CsMADSO1 during flower and fruit development in cucumber. (A and B) RT-PCR analysis of CsMADSO1 in five different developmental stages (MF1 to MF5, and FF1 to FF5) in cucumber. (C) In silico expression analysis of CSMADSO1 during fruit development in cucumber. The expression of CsMADSO1 is presented as RPKM values. 
1174

A
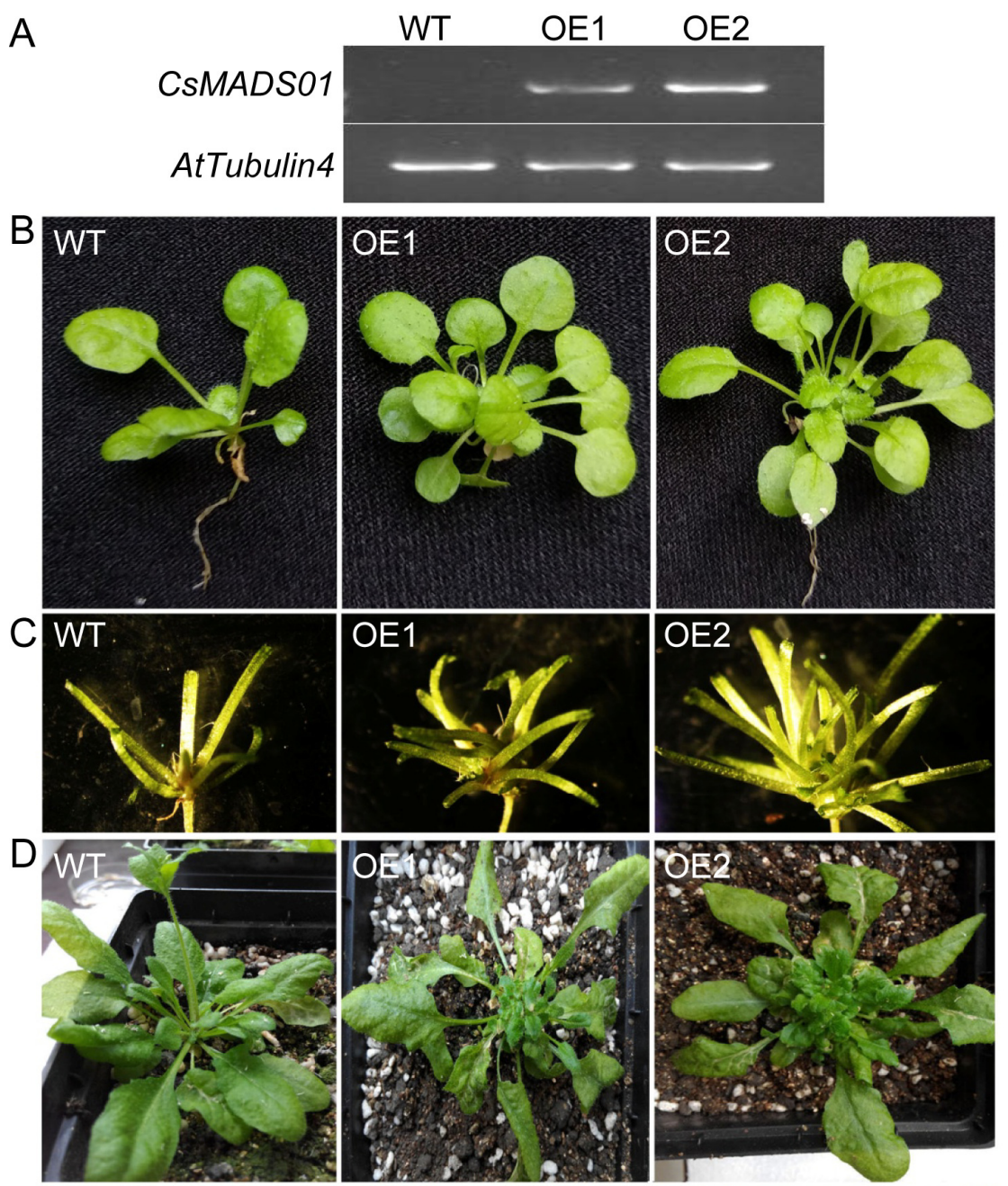

Fig. 6. Overexpression of CsMADSO1 in Arabidopsis. (A) RT-PCR analysis of CsMADSO1 in WT and transgenic lines (OE1 and $\mathrm{OE} 2)$. (B and C) Phenotypes of CsMADS01 transgenic lines (OE1 and OE2) and WT plants at 22 days after sowing. (B) Overexpression of CsMADSOI caused a leafy phenotype. (C) The branches of transgenic Arabidopsis and WT plants were shown. (D) Phenotypes of CsMADSO1 transgenic lines (OE1 and OE2) and WT plants at 36 days after sowing

repressor to influence grain yield by suppressing the transcription of related genes (Ren $e t$ al., 2016). The sterile lemmas, lemmas, paleas, and the inner floral organs were converted into leaf-like structures in the osmadsl-z osmads5-3 osmads34-1 triple mutant (Wu et al., 2018). A recent study showed that compared with Rosa chinensis cv. Old Blush, $R$. chinensis cv. Viridiflora displayed a phyllody phenotype, in which flower organs were converted into leaflike organs, and this phenomenon is associated with the modified expression of the genes related to flowering and flower development, including RcSEP1 and RcSEP3 (Yan et al., 2016). Therefore, it can be speculated that the phyllodylike phenotype of CsMADSOI-overexpressing transgenic Arabidopsis plants may be due to the misexpression of Arabidopsis endogenic SEPs and other flower organ identity genes. Similarly, ectopic expression of a wheat $S E P$-like gene, TaMADS1, caused early flowering and abnormal development of all floral organs in Arabidopsis, including the conversion of sepals into leaf-like structures, which may be due to the regulation of some flower meristem identity genes (Zhao et al., 2006).

\section{Conclusions}

In summary, we performed the isolation and functional characterization of CSMADSO1 from cucumber. This gene was previously named as CSSEP 2 by a previous study (Wang et al., 2016), and is involved in floral organ and fruit development. The deduced CsMADS01 protein contains the typical MADS, I, K, and C domains, as well as two conserved motifs (SEP I and SEP II) specific for SEP proteins. Overexpression of CsMADSO1 in Arabidopsis led to a phyllody-like phenotype and the transgenic plants never flowered. These results lay a foundation for further revealing the regulatory mechanisms of flower and fruit development in cucumber. 


\section{Acknowledgements}

This work was funded by the National Natural Science Foundation of China (31660578), and the NSFC-Henan Province Joint Foundation (U1204307), Program for Tackling Key Problems in Science and Technology of Henan Province (152102210334), Key Research Projects of Henan Higher Education Institutions (16A180030).

\section{Conflict of Interest}

The authors declare that there are no conflicts of interest related to this article.

\section{References}

Alvarez-Buylla ER, Pelaz S, Liljegren SJ, Gold SE, Burgeff C, Ditta GS, ... Yanofksy MF (2000). An ancestral MADS-box gene duplication occurred before the divergence of plants and animals. Proceedings of the National Academy of Sciences of the United States of America 97(10):5328-5333.

Angenent GC,Franken J, Busscher M, Weiss D, van Tunen AJ (1994).Cosuppression of the petunia homeotic gene $f b p 2$ affects the identity of the generative meristem. The Plant Journal 5(1):33-44.

Arora R, Agarwal P, Ray S, Singh AK, Singh VP, Tyagi AK, Kapoor S (2007). MADS-box gene family in rice: genome-wide identification, organization and expression profiling during reproductive development and stress. BMC Genomics 8(1):242.

Chen R, Ma J, Luo D, Hou X, Ma F, Zhang Y, ... Guo W (2019). CaMADS, a MADS-box transcription factor from pepper, plays an important role in the response to cold, salt, and osmotic stress. Plant Science 280:164174.

ChengSY, ChengJH, FengX, Ye JB, WangXH (2016). Molecular cloning and expression analysis of a putative $\mathrm{E}$ class MADS-box gene, GbSEP, from Ginkgo biloba. Journal of Animal and Plant Sciences 26(1):253260.

Clough SJ, Bent AF (1998). Floral dip: a simplified method for Agrobacterium-mediated transformation of Arabidopsis thaliana. The Plant Journal 16(6):735-743.

Cui R, Han J, Zhao S, Su K, Wu F, Du X, ... Meng Z (2010). Functional conservation and diversification of class $\mathrm{E}$ floral homeotic genes in rice (Oryzasativa). The Plant Journal 61(5):767-781.

Ditta G, Pinyopich A, Robles P, Pelaz S, Yanofsky MF (2004). The SEP4 gene of Arabidopsis thaliana functions in floral organ and meristem identity. Current Biology 14(21):1935-1940.

Gao X, Liang W, Yin C, Ji S, Wang H, Su X, ... Zhang D (2010). The SEPALLATA-like gene OsMADS34 is required for rice inflorescence and spikelet development. Plant Physiology 153(2):728-740.

Gutierrez-Cortines ME, Davies B (2000). Beyond the ABCs: ternary complex formation in the control of floral organ identity. Trends in Plant Science 5(11):471-476.

Honma T, Goto K (2001). Complexes of MADS-box proteins are sufficient to convert leaves into floral organs. Nature 409(6819):525529.

Hu L, Liu S (2012). Genome-wide analysis of the MADS-box gene family in cucumber.Genome 55(3):245-256.
Hu Y,Liang W, Yin C, YangX, Ping B,Li A, ... Yuan Z(2015).Interactions of $O s M A D S I$ with floral homeotic genes in rice flower development. Molecular Plant 8(9):1366-1384.

Ireland HS, Yao JL, Tomes S, Sutherland PW, Nieuwenhuizen N, Gunaseelan K, ... Schaffer RJ (2013). Apple SEPALLATA1/2-like genes control fruit flesh development and ripening. The Plant Journal 73(6):10441056.

Ito Y,Nishizawa-Yokoi A, Endo M, Mikami M, Shima Y, Nakamura N, ... TokiS (2017). Re-evaluation of the rin mutation and the role of RIN in the induction of tomato ripening. Nature Plants3(11):866-874.

Kaufmann K, Melzer R, Theissen G (2005). MIKC-type MADS-domain proteins: structural modularity, protein interactions and network evolution in land plants. Gene 347(2):183-198.

Khanday I, Yadav SR, Vijayraghavan U (2013). Rice LHS1/OsMADS1 controls floret meristem specification by coordinated regulation of transcription factors and hormone signaling pathways. Plant Physiology 161(4):1970-1983.

Kobayashi K, Maekawa M, Miyao A, Hirochika H, Kyozuka J (2010). PANICLE PHYTOMER2 (PAP2), encoding a SEPALLATA subfamily MADS-box protein, positively controls spikelet meristem identity in rice. Plant and Cell Physiology 51(1):47-57.

Kobayashi K, Yasuno N, Sato Y, Yoda M, Yamazaki R, Kimizu M, ... Kyozuka J (2012). Inflorescence meristem identity in rice is specified by overlapping functions of three $A P I / F U L$-like MADS box genes and PAP2, a SEPALLATA MADS box gene. The Plant Cell 24(5):18481859.

Li J, Li F, Qian M, Han M, Liu H, Zhang D, ... Zhao C (2017). Characteristics and regulatory pathway of the PrupeSEPI SEPALLATA gene during ripening and softening in peach fruits. Plant Science 257:63-73.

Li S, Chen K, Grierson D (2019). A critical evaluation of the role of ethylene and MADS transcription factors in the network controlling fleshy fruit ripening. New Phytologist 221(4):17241741.

Li S, Xu H, Ju Z, Cao D, Zhu H, Fu D, ... Zhu B (2018). The RIN-MC Fusion of MADS-box transcription factors has transcriptional activity and modulates expression of many ripening genes. Plant Physiology 176(1):891-909.

Lin X, Wu F, Du X, Shi X, Liu Y,Liu S, ... MengZ (2014). The pleiotropic SEPALLATA-like gene OsMADS34 reveals that the 'empty glumes' of rice (Oryza sativa) spikelets are in fact rudimentary lemmas. New Phytologist 202(2):689-702.

Lü P, Yu S, Zhu N, Chen YR, Zhou B, Pan Y, ...Z ZhongS (2018). Genome encode analyses reveal the basis of convergent evolution of fleshy fruit ripening, Nature Plants 4(10):784791.

Luo Y, Li H, Xiang Z, He N (2018). Identification of Morus notabilis MADS-box genes and elucidation of the roles of MnMADS33 during endodormancy.Scientific Reports 8(1):5860.

Maejima K, Iwai R, Himeno M, Komatsu K, Kitazawa Y, Fujita N, ... Namba $S$ (2014). Recognition of floral homeotic MADS domain transcription factors by a phytoplasmal effector, phyllogen, induces phyllody. The Plant Journal 78(4):541-554.

Maejima K, Kitazawa Y, Tomomitsu T, Yusa A, Neriya Y, Himeno M, ... Namba S (2015). Degradation of class E MADS-domain transcription factors in Arabidopsis by a phytoplasmal effector, phyllogen. Plant Signaling\& Behavior 10(8):e1042635. 
1176

Malcomber ST, Kellogg EA (2005). SEPALLATA gene diversification: brave new whorls. Trends in Plant Science 10(9):427-435.

Masiero S, Colombo L, Grini PE, Schnittger A, Kater MM (2011). The emerging importance of type I MADS box transcription factors for plant reproduction. The Plant Cell 23(3):865-872.

Meng Q, Li X, Zhu W, Yang L, Liang W, Dreni L, Zhang D (2017). Regulatory network and genetic interactions established by OsMADS34 in rice inflorescence and spikelet morphogenesis. Journal of Integrative Plant Biology 59(9):693-707.

Mitoma M, Kanno A (2018). The greenish flower phenotype of Habenaria radiata (Orchidaceae) is caused by a mutation in the SEPALLATA-like MADS-box gene HrSEP-1. Frontiers in Plant Science 9:831.

NardeliSM, ArticoS, Aoyagi GM, de Moura SM, da Franca Silva T, Grosside-Sa MF, ... Alves-Ferreira M (2018). Genome-wide analysis of the MADS-box gene family in polyploid cotton (Gossypium hirsutum) and in its diploid parental species (Gossypium arboreum and Gossypium raimondii). Plant Physiology and Biochemistry 127:169-184.

Pelaz S, Ditta GS, Baumann E, Wisman E, Yanofsky MF (2000). B and C floral organ identity functions require SEPALLATA MADS-box genes. Nature 405(6783):200-203.

Pelaz S, Gustafson-Brown C, Kohalmi SE, Crosby WL, Yanofsky MF (2001). APETALAI and SEPALLATA3 interact to promote flower development. The Plant Journal26(4):385-394.

Pnueli L, Hareven D, Broday L, Hurwitz C, Lifschitz E (1994). The TM5 MADS box gene mediates organ differentiation in the three inner whorls of tomato flowers. The Plant Cell 6(2):175-186.

Ren D, Rao Y, Leng Y, Li Z, Xu Q, Wu L, ... Qian Q (2016). Regulatory role of $O S M A D S 34$ in the determination of giumes fate, grain yield, and quality in rice. Frontiers in PlantScience 7:1853.

RenZ, YuD, YangZ,LiC, Qanmber G,LiY,...LiF (2017). Genome-wide identification of the MIKC-type MADS-box gene family in Gossypium birsutum L. unravels their roles in flowering. Frontiers in Plant Science $8: 384$.

Riechmann JL, Meyerowitz EM (1997). MADS domain proteins in plant development. Biological Chemistry378(10):1079-1101.

Seymour GB, Ryder CD, Cevik V, Hammond JP, Popovich A, King GJ, ... Manning K (2011). A SEPALLATA gene is involved in the development and ripening of strawberry (Fragaria $\mathrm{x}$ ananassa Duch.) fruit, a non-climacteric tissue. Journal of Experimental Botany 62(3):1179-1188.

Sheng XG, Zhao ZQ, Wang JS, Yu HF, Shen YS, Zeng XY, Gu HH (2019). Genome wide analysis of MADS-box gene family in Brassica oleracea reveals conservation and variation in flower development. BMC Plant Biology 19(1):106.

Soza VL, Snelson CD, Hewett Hazelton KD, Di Stilio VS (2016). Partial redundancy and functional specialization of E-class SEPALLATA genes in an early-diverging eudicot. Developmental Biology 419(1):143155.

Theissen G, Melzer R, Rumpler F (2016). MADS-domain transcription factors and the floral quartet model of flower development: linking plant development and evolution. Development 143(18):3259-3271.

Theissen G, Saedler H (2001). Plant biology. Floral quartets. Nature 409(6819):469-471.

Tzeng TY, Hsiao CC, Chi PJ, Yang CH (2003). Two lily SEPALLATA- like genes cause different effects on floral formation and floral transition in Arabidopsis. Plant Physiology 133(3):1091-1101.

Wang X, Gao D, Sun J, Liu M, Lun Y, Zheng J, ... Huang S (2016). An exon skippingin a SEPALLATA-Like gene is associated with perturbed floral and fruits development in cucumber. Journal of Integrative Plant Biology 58(9):766-771.

Weigel D, MeyerowitzEM(1994). The ABCs offloral homeotic genes. Cell 78(2):203-209.

Wu D, Liang W, Zhu W, Chen M, Ferrandiz C, Burton RA, ... Zhang D (2018). Loss of LOFSEP transcription factor function converts spikelet to leaf-like structures in rice.Plant Physiology 176(2):1646-1664.

Xu Y, Zhang L, Xie H, Zhang YQ, Oliveira MM, Ma RC (2008). Expression analysis and genetic mapping of three SEPALLATA-like genes from peach (Prunus persica (L.) Batsch). Tree Genetics \& Genomes 4(4):693-703.

Xu Z, Zhang Q, Sun L, Du D, Cheng T, Pan H, ... Wang J (2014). Genome-wide identification, characterisation and expression analysis of the MADS-box gene family in Prunus mume. Molecular Genetics and Genomics 289(5):903-920.

Yan H, Zhang H, Wang Q, Jian H, Qiu X, Baudino S, ... Tang K (2016). The Rosa chinensis cv. Viridiflora phyllody phenotype is associated with misexpression of flower organ identity genes. Frontiers in Plant Science 7:996.

Zahn LM, Feng B, Ma H (2006). Beyond the ABC-model: regulation of floral homeotic genes. Advances in Botanical Research 44(06):163-207.

Zahn LM, Kong H, Leebens-Mack JH, Kim S, Soltis PS, Landherr LL, ... Ma H (2005). The evolution of the SEPALLATA subfamily of MADS-box genes: a preangiosperm origin with multiple duplications throughout angiosperm history. Genetics 169(4):2209-2223.

Zhang L, Zhao J, Feng C, Liu M, Wang J, Hu Y (2017a). Genome-wide identification, characterization of the MADS-box gene family in Chinese jujube and their involvement in flower development. Scientific Reports 7(1):1025.

Zhang S, Lu S, Yi S, Han H, Liu L, Zhang J, ... Liu G (2017b). Functional conservation and divergence of five SEPALLATA-like genes from a basal eudicot tree, Platanus acerifolia. Planta 245(2):439-457.

Zhao XY, Cheng ZJ, Zhang XS (2006). Overexpression of TaMADS1, a SEPALLATA-like gene in wheat, causes early flowering and the abnormal development of floral organs in Arabidopsis. Planta 223(4):698-707.

Zhou Y, Ge L, Hu L, Yang Y, Liu S (2018). A cucumber AGAMOUSLIKE 15 (AGL15) MADS-box gene mediates abnormal leat morphology in Arabidopsis. Agronomy 8(11):265.

Zhou Y, Hu L, Song J, Jiang L, Liu S (2019a). Isolation and characterization of a MADS-box gene in cucumber (Cucumis sativus L.) that affects flowering time and leaf morphology in transgenic Arabidopsis. Biotechnology \& Biotechnological Equipment 33(1):5463.

Zhou Y, Hu L, Ye S, Jiang L, Liu S (2019b). Overexpression of an APETALA1-like gene from cucumber (Cucumis sativus $\mathrm{L}$.) induces earlier flowering and abnormal leaf development in transgenic Arabidopsis. Canadian Journal of Plant Science 99(2):210-220.

Zhou Y, Xu Z, Yong X, Ahmad S, Yang W, Cheng T, ... Zhang Q (2017). SEP-class genes in Prunus mume and their likely role in floral organ development. BMC Plant Biology 17(1):10. 
Table S1. RT-PCR primers used for gene expression analysis in this study

\begin{tabular}{ccc}
\hline Gene & Forward primer & Reverse primer \\
\hline CsMADSO1 & AGAAACCTTCTTGGGGAGGA & GAAGCCATTGACTTGTTGGG \\
CsAct3 & GACATTCAATGTGCCTGCTATG & CATACCGATGAGAGATGGCTG \\
AtTubulin4 & GCGAACAGTTCACAGCTATGTTCA & GAGGGAGCCATTGACAACATCTT \\
\hline
\end{tabular}

